\title{
Positronium formation during scattering of positrons by hydrogen atoms
}

\author{
Sultana N. Nahar \\ Department of Physics and Astronomy, Georgia State University, Atlanta, Georgia 30303 \\ (Received 20 July 1989)
}

The formation of positronium (Ps) in different states by the impact of intermediate energy (20-500 eV) positrons on atomic hydrogen is calculated using the distorted-wave Born approximation along with the first Born approximation. Differential and integrated cross sections for the formation of $\operatorname{Ps}(1 s), \operatorname{Ps}(2 s), \operatorname{Ps}\left(2 p_{0}\right)$, and $\operatorname{Ps}\left(2 p_{1}\right)$ have been evaluated individually and then $1 / n^{3}$ behavior for charge-transfer cross sections is used for $n \geq 3$ to obtain the total cross sections for positronium formation. The present results for positronium formation cross sections are in agreement with the results of other available investigations.

\section{INTRODUCTION}

After realizing the possibility of positronium (Ps) formation ${ }^{1}$ by capture of an electron by a positron before they annihilate each other, the first calculation of positronium formation was carried out in $1954 .^{2}$ Later, experimental evidences for the formation of Ps in $n=2$ excited states by Verghese et $a l .{ }^{3}$ in $2 p$ excited states by Canter et al., 4 and energy splitting of the first excited states of Ps by Mills et al. and Hatamian et al. ${ }^{5}$ were found. (For additional references see the review article by Stroscio. ${ }^{6}$ ) In the present work the cross sections for the formation of positronium from atomic hydrogen by the impact of intermediate-energy positrons have been calculated by using the distorted-wave Born approximation (DWBA) along with the first Born approximation (FBA). The cross sections for $\operatorname{Ps}(1 s), \operatorname{Ps}(2 s), \operatorname{Ps}\left(2 p_{0}\right)$, and $\operatorname{Ps}\left(2 p_{1}\right)$ formations are obtained individually and then the $1 / n^{3}$ behavior for charge-transfer cross sections is used for $n \geq 3$ to obtain the total cross sections for positronium formation.

In the first Born calculations for the capture process in proton-hydrogen collisions done by Oppenheimer ${ }^{7}$ and by Brinkman and Kramers, ${ }^{B}$ the calculated cross sections were several times larger than the measured ones. In these calculations the repulsive interaction between the projectile and the target nucleus was neglected as it causes only the deflection of the projectile trajectory, which depends on the mass and energy of the projectile, but no change in the internal state of the target atom. Results were improved significantly by including this projectile-target core interaction. In the Born calculation for capture cross sections, this inclusion requires evaluation of additional terms which are important especially for heavy projectiles at very large impact energies. As an alternative to the Born series, the distorted-wave Born approximation ${ }^{9.10}$ has been used in the present work to obtain the positronium formation cross sections and the form of DWBA used is that of Ref. 10 .

\section{THEORY}

The method and formulations of calculating the cross sections for positronium formation from hydrogen in the FBA and DWBA used in the present work are similar to those of Ref. 10. $M_{p}, m$, and $M_{T}$ are the projectile, the atomic electron, and the proton mass, respectively. The projectile-atomic-electron interaction $V_{p-e}$, the projectile-proton interaction $V_{p}(R)$, and the distortion potential $U_{i}\left(\mathbf{R}_{T}\right)$ (Ref. 10) in the initial channel are (in a.u.)

$$
\begin{aligned}
& V_{p-e}\left(r_{p}\right)=-1 / r_{p}, \quad V_{p}(R)=1 / R, \\
& U_{i}\left(\mathbf{R}_{T}\right)=-U_{1}\left(\mathbf{R}_{T}\right)+V_{p}\left(\mathbf{R}_{T}\right),
\end{aligned}
$$

where

$$
U_{i}\left(\mathbf{R}_{T}\right)=\int\left|\phi_{i}\left(\mathbf{r}_{T}\right)\right|^{2} \frac{1}{\left|\mathbf{r}_{T}-\mathbf{R}_{T}\right|} d^{3} r
$$

$\phi_{i}\left(\mathbf{r}_{T}\right)$ is the hydrogen wave function. The definitions of different position vectors are shown in Fig. 1. In the final channel, the electron-bare-proton interaction $V_{p-T}\left(r_{r}\right)$, positron-bare-proton interaction $V_{p}(R)$, and the distortion potential $U_{f}$ are

$$
V_{p-T}\left(r_{T}\right)=-1 / r_{T}, \quad V_{p}(R)=1 / R, \quad U_{f}\left(\mathbf{R}_{p}\right)=0 .
$$

The distortion potential $U_{f}$ in the final channel vanishes because of the value of $\beta=M_{p} /\left(m+M_{p}\right)=\frac{1}{2} .{ }^{10}$ The present work is an extension of work by Shakeshaft and Wadehra ${ }^{10}$ with the difference that the calculations have

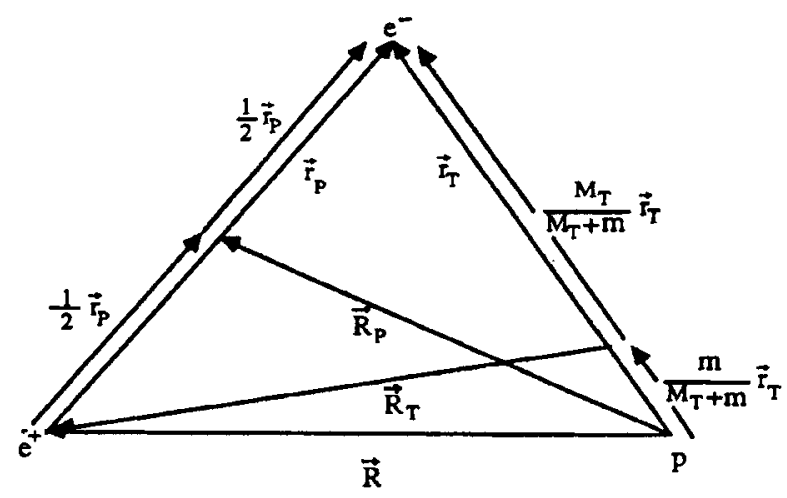

FIG. 1. The coordinate system. 
been carried out in the prior form of the process instead of in the post form of the process. The extension has been made to the calculations of $\operatorname{Ps}(1 s), \operatorname{Ps}(2 s)$, and $\operatorname{Ps}(2 p)$ formations in the post and the prior form using the FBA and in the prior form using the DWBA and to the total cross section for Ps formation using the $1 / n^{3}$ behavior of the charge-transfer cross section.

For the formation of $\operatorname{Ps}(2 s)$, the transition matrix element $T$ is calculated easily by writing the Fourier transform of the final Ps wave function, $\tilde{\phi}_{f}(\mathbf{k})$, in a similar way to $\mathrm{Ps}(1 s)$ formation as $\widetilde{\phi}_{f}(\mathbf{k})=-\frac{16}{\sqrt{2 \pi}}\left(\frac{1}{2 a_{\mathrm{Ps}}}\right)^{5 / 2} Y_{00} \sum_{i=1}^{2} f_{i} \frac{d^{i}}{d u^{i}} \frac{1}{u+k^{2}}$,

where $a_{\mathrm{Ps}}$ is the Bohr radius for positronium (equal to 2 a.u.), $f_{1}=1, f_{2}=2 u$, and $u=\left(1 / 2 a_{\mathrm{Ps}}\right)^{2}$. The $p$-state wave functions of positronium, unlike spherically sym. metric s-state wave functions, are angle dependent and introduce more complex calculations for capture cross sections. The complexity can be reduced" by expressing the angular dependence of the wave functions in terms of derivatives of an exponential factor as follows:

$$
\begin{aligned}
& \phi_{f}(r)=R_{21}(r) Y_{10}(p)=\frac{1}{\sqrt{\pi}}\left[\frac{1}{2 a_{\mathrm{Ps}}}\right]^{s / 2} e^{-r / 2 a_{\mathrm{Ps}}} r \cos \theta=i \frac{\partial}{\partial \chi_{z}}\left[\phi_{f}^{\prime}(r) \exp (-i \chi \cdot r)\right]_{\chi=0}, \\
& \phi_{f}(r)=R_{21}(r) Y_{1-1}(\gamma)=\frac{1}{\sqrt{2 \pi}}\left(\frac{1}{2 a_{\mathrm{Ps}}}\right)^{5 / 2} e^{-r / 2 a_{\mathrm{Ps}}} r(\sin \theta) e^{-i \phi}=\frac{i}{\sqrt{2}} \int \frac{\partial}{\partial \chi_{x}}\left[\phi_{f}^{\prime}(r) \exp (-i \chi \cdot r)\right] \\
& \left.-i \frac{\partial}{\partial \chi_{y}}\left[\phi_{f}^{\prime}(r) \exp (-i \chi \cdot r)\right]\right]_{\chi=0} \\
& \phi_{f}(r)=R_{21}(r) Y_{11}(\hat{r})=-\frac{1}{\sqrt{2 \pi}}\left(\left.\frac{1}{2 a_{\mathrm{Ps}}}\right|^{S / 2} e^{-r / 2 a_{\mathrm{Ps}}} r(\sin \theta) e^{i \phi}=-\frac{i}{\sqrt{2}}\left\lceil\frac{\partial}{\partial \chi_{x}}\left[\phi_{f}^{\prime}(r) \exp (-i \chi \cdot r)\right]\right.\right. \\
& +\left.i \frac{\partial}{\partial \chi_{y}}\left[\phi_{f}^{\prime}(r) \exp (-i \chi \cdot r)\right]\right|_{\chi=0} \text {, }
\end{aligned}
$$

where

$$
\phi_{f}^{\prime}(r)=\frac{1}{\sqrt{\pi}}\left(\frac{1}{2 a_{\mathrm{Ps}}}\right)^{5 / 2} \exp \left[-\frac{r}{2 a_{\mathrm{Ps}}}\right] .
$$

Then the various terms in the $T$-matrix element, $T=T_{1}+T_{2}$ (see Appendixes of Ref. 10), for the formation of, for example, $\operatorname{Ps}\left(2 p_{0}\right)$ in the prior form of the DWBA, can be expressed as

$$
T_{1}=N_{2}+N_{3}
$$

where

$$
\begin{aligned}
N_{2}=-i(2 \pi)^{3 / 2} \frac{\partial}{\partial \chi_{z}}[ & {\left[\frac{K^{2}}{2 \mu_{\rho}}-\varepsilon_{f}\right] \bar{\phi}_{f}^{\prime *} } \\
& \left.\times(\mathbf{K}+\chi) \bar{\phi}_{i}(-\mathbf{J})\right]_{\chi=0}, \\
N_{3}=i(2 \pi)^{3 / 2} \frac{\partial}{\partial \chi_{z}}\left[\int \bar{\phi}_{f}^{\prime *}(\mathbf{K}+\chi-\mathbf{t}) \breve{U}_{1}(\mathrm{t})\right. & \\
& \left.\times \bar{\phi}_{i}(-\mathrm{J}-\alpha \mathrm{t}) d^{3} t\right]_{\chi=0},
\end{aligned}
$$

and

$$
T_{2}=L_{2}+L_{3},
$$

where

$$
\begin{aligned}
& L_{2}=-i(2 \pi)^{3 / 2} \frac{\partial}{\partial \chi_{z}}\left\lfloor\int \tilde{\phi}_{f}^{\prime *}\left(\beta \mathbf{k}_{f}+\chi-\mathbf{q}\right)\right. \\
& \times\left[\frac{\left(\beta \mathbf{k}_{f}+\chi-\mathrm{q}\right)^{2}}{2 \mu_{\mathrm{Ps}}}-\varepsilon_{f}\right] \\
&\left.\times \boldsymbol{g}_{i}^{+}(\mathbf{q}) \tilde{\phi}_{i}\left(\mathbf{k}_{f}-\alpha \mathrm{q}\right) d^{3} q\right]_{\chi=0},
\end{aligned}
$$

$$
\begin{array}{r}
L_{3}=i \frac{\partial}{\partial \chi_{z}} \iint \widetilde{\phi}_{f}^{* *}\left(\beta \mathbf{k}_{f}+\chi-\mathbf{t}-\mathbf{q}\right) \widetilde{U}_{1}(\mathbf{t}) \tilde{g}_{i}^{+}(\mathbf{q}) \\
\quad \times\left.\tilde{\phi}_{i}\left(\mathbf{k}_{f}-\alpha \mathbf{t}-\alpha \mathbf{q}\right) d^{3} t d^{3} q\right|_{\chi=0}
\end{array}
$$

$\mu_{\mathrm{Ps}}$ and $\varepsilon_{f}$ are the reduced mass and the internal energy of the final bound-atom positronium, $\alpha=M_{T} /\left(m+M_{T}\right)$, $v_{i}$ and $v_{f}$ are the reduced masses in the initial channel and the final channel, respectively, $k_{i}$ and $k_{f}$ are the initial and final relative momenta, $\mathbf{K}=\beta \mathbf{k}_{f}-\mathbf{k}_{i}$ and $\mathbf{J}=\alpha \mathbf{k}_{i}-\mathbf{k}_{f}$ in Eqs. (7a) and (7b) are the momentum transfer vectors, and the Fourier transform of the distortion factor $g_{i}$ is given by ${ }^{10}$

$$
\tilde{g}_{i}^{+}(\mathbf{K})=-\frac{2 v_{i}}{\hbar^{2}} \frac{\widetilde{U}_{i}\left(\mathbf{K}-\mathbf{k}_{i}\right)}{K^{2}-k_{i}^{2}-i \eta},
$$

where $\eta \rightarrow 0$. In the post form of the FBA, the $T$-matrix element for the formation of $\operatorname{Ps}\left(2 p_{0}\right)$ is given by

$$
T=N_{1}+N_{2 p}
$$


where

$$
\begin{array}{r}
N_{1}=i(2 \pi)^{3 / 2} \frac{\partial}{\partial \chi_{z}}\left[\int \tilde{\phi}_{f}^{\prime *}(\mathbf{K}+\chi-\mathrm{t}) \tilde{V}_{p}(\mathrm{t})\right. \\
\left.\quad \times \tilde{\phi}_{i}(-\mathrm{t}-\mathrm{J}) d^{3} t\right]_{\chi=0}, \\
\begin{array}{r}
N_{2 p}=i(2 \pi)^{3 / 2} \frac{\partial}{\partial \chi_{z}}\left[\tilde{\phi}_{f}^{\prime *}(\mathbf{K}+\chi)\right. \\
\quad \times \int \tilde{V}_{p-r}(\mathrm{t}) \\
\quad \times\left.\tilde{\phi}_{i}(-\mathrm{t}-\mathrm{J}) d^{3} t\right|_{\chi=0},
\end{array}
\end{array}
$$

and, in the prior form of the FBA,

$$
T=N_{1}+N_{2}
$$

where $N_{1}$ and $N_{2}$ have been defined in Eqs. (10a) and (7a). Now, these various terms of the $T$-matrix elements can be evaluated, after substitution of the explicit forms of the functions, by reducing the relevant integrals to one- or two-dimensional integrals using the Feynman identity as described in Ref. 10. The $T$-matrix elements for $\operatorname{Ps}\left(2 p_{1}\right)$ or $\operatorname{Ps}\left(2 p_{-1}\right)$ formations are evaluated in a similar manner. The differential cross section is obtained as

$$
\frac{d \sigma}{d \Omega}=\frac{v_{i} v_{f}}{(2 \pi)^{2}} \frac{k_{f}}{k_{i}}|T|^{2},
$$

where in the case of the DWBA, terms up to the third order of potential are kept in the expansion of $|T|^{2}$. The integrated cross section is given by

$$
\sigma=2 \pi \int_{0}^{\pi}\left[\frac{d \sigma}{d \Omega}\right] \sin \theta d \theta
$$

which is evaluated numerically.

It was predicted by Oppenheimer ${ }^{7}$ that for capture into any $n s$ excited state at high incident energies, the cross section falls as $1 / n^{3}$. Later Omidvar ${ }^{12}$ showed that the cross section for capture into the $s, p$, and $d$ states as well as sum of the cross sections over all the angular momentum states (i.e., sum over $l$ and $m$ ) falls as $1 / n^{3}$ in the first Born approximation. There is also an experimental evidence of cross section for electron capture into excited states falling as $1 / n^{3}$ in collisions of flourine ions in argon gas. ${ }^{13}$ Hence, using the $1 / n^{3}$ dependence of the capture cross section for the formation of Ps in excited states $(n \geq 3)$, the total cross section could be written as

$$
\begin{gathered}
\sigma^{r}=\sigma^{1}+\sigma^{2}\left[1+\left[\frac{n}{n+1}\right]^{3}+\left[\frac{n}{n+2}\right]^{3}\right. \\
\left.+\left[\frac{n}{n+3}\right]^{3}+\cdots\right]
\end{gathered}
$$

where $\sigma^{1}=\sigma(1 s), \quad \sigma^{2}=\sigma(2 s)+\sigma\left(2 p_{0}\right)+2 \sigma\left(2 p_{1}\right), \quad$ and $n=2$. The value of the series within the large square brackets of Eq. (13) with $n=2$ is 1.616455 .

\section{RESULTS AND DISCUSSION}

The differential cross sections (DCS's) for the positronium formation in all bound states using both the FBA and DWBA are shown in Figs. 2-5. The general feature of the present DCS curves using the DWBA is similar to many other investigations showing a large peak in the forward direction followed by a minimum and then decaying smoothly at larger scattering angles. This minimum in the DWBA calculation is at an angle different from that of the FBA. Similar to the previous calculation ${ }^{10}$ for $\mathrm{Ps}(1 s)$ formation from a hydrogen atom, the differential cross section in the DWBA is found to be negative at larger angles at most of the impact energies considered and at the minimum at higher energies. At the lower energies considered $(20-60 \mathrm{eV})$, the total differential cross section (that is, after summing up the cross sections for all states formation) stayed positive throughout the complete scattering angular range. At somewhat higher positron energies $(75-125 \mathrm{eV})$, the value of the DCS becomes negative at some higher scattering angle and then becomes positive again. The negative values for DCS's resulted from the dominance of

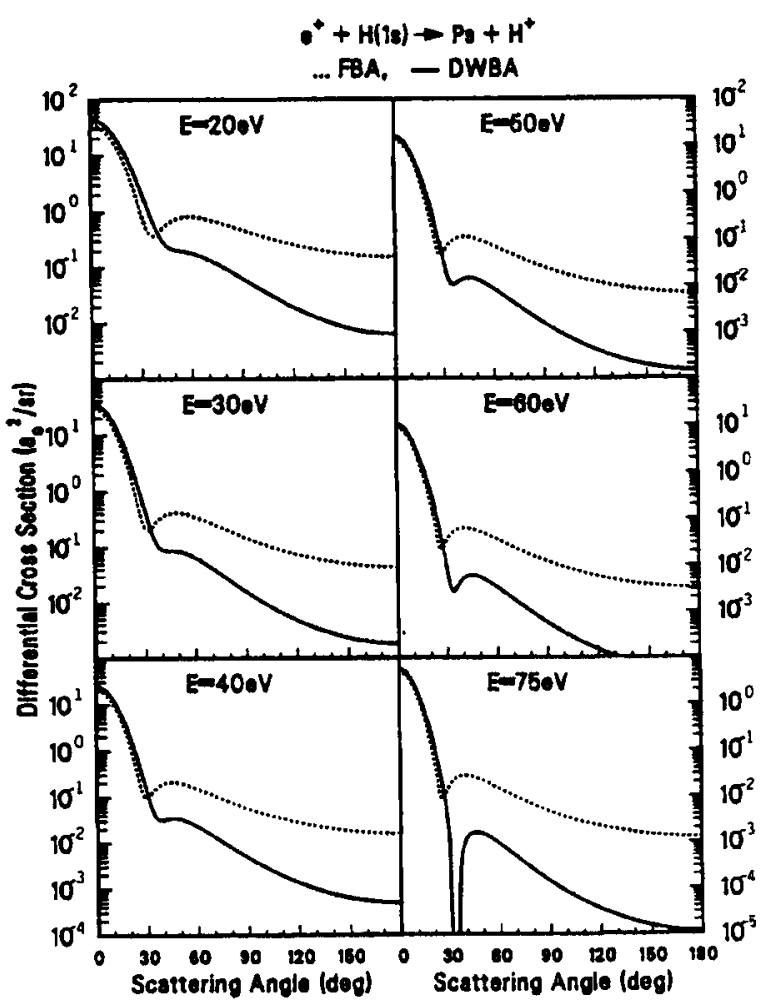

FIG. 2. Total (all $n, l, m$ ) differential cross sections for positronium formation from hydrogen atoms at various positron impact energies. 
the positron-electron interaction (term $N_{2}$ ) which is attractive in nature and has a slow decrease with larger angles in the $T$-matrix element. This negative contribution could have been compensated by keeping fourth- or higher-order terms in potential in the evaluation of the $T$ matrix. In that case, the Thomas peak in the DCS curves probably could have been seen at even higher positron energies than were observed by McGuire et al. ${ }^{14}$ and Basu and Ghosh ${ }^{15}$ in their calculations of ground-state Ps formation using the Born approximation up to second order. However, for the integrated cross sections the contributions from the higher scattering angles at these energies are very small, as noted in the present work as well as in other investigations.

The integrated cross sections for the formation of $\operatorname{Ps}(1 s), \operatorname{Ps}(2 s), \operatorname{Ps}\left(2 p_{0}\right)$, and $\operatorname{Ps}\left(2 p_{1}\right)$ calculated in the prior form using both approximations, the FBA and DWBA, are presented in Table $I$. In FBA cross sections, the post-prior discrepancy is found to be negligibly small; the difference between the post and the prior values of the cross sections being one part in $10^{4}$ or $10^{5}$. Hence, only the prior-form values are presented in Table I. There are some differences between the present cross sections for Ps(1s) formation and those of Ref. 10. The present values are larger than those of Ref. 10. The reason is that the cross sections evaluated in Ref. 10 were not integrated up to $180^{\circ}$ of scattering angle and a small discrepancy is introduced by the assumption $\alpha=1$ in the post and prior terms. Some of the other investigations for positronium formation have been made by Cheshire ${ }^{16}$ using the first Born and the impulse approximations, Chen and Kra-

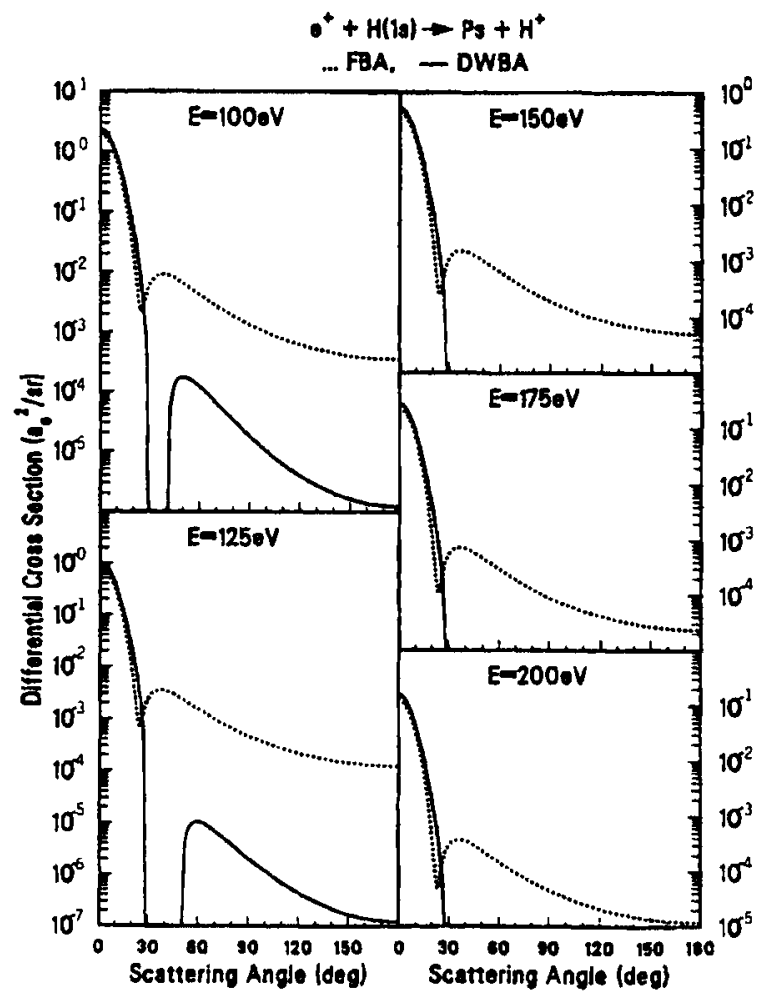

FIG. 3. Same as Fig. 2.

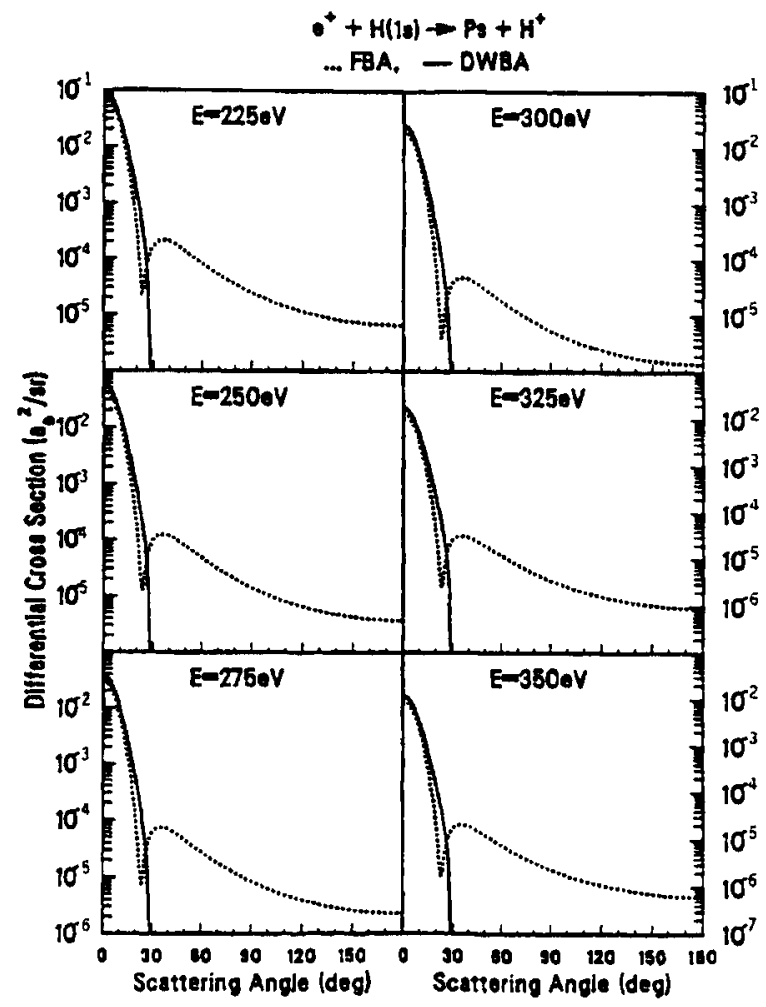

FIG. 4. Same as Fig. 2.

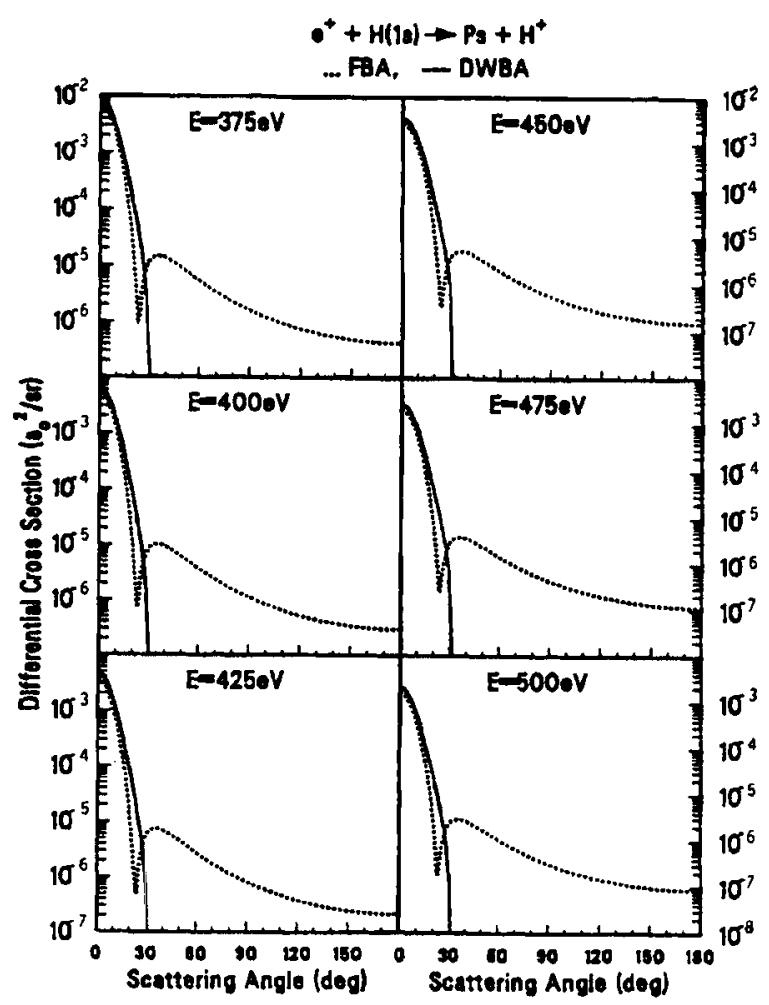

FIG. 5. Same as Fig. 2. 
TABLE 1. The integrated cross sections (in units of $a_{0}^{2}$ ) using the FBA and DWBA for the formation of positronium in the states $1 s, 2 s, 2 p_{0}$, and $2 p_{1}$ from a hydrogen atom by the impact of positrons. All the cross sections are calculated in the prior form. The notation $a[b]$ means $a \times 10^{b}$.

\begin{tabular}{|c|c|c|c|c|c|c|c|c|}
\hline \multirow{3}{*}{$\begin{array}{c}E \\
(\mathrm{eV}) \\
\end{array}$} & \multicolumn{6}{|c|}{ Cross sections $\left(a_{0}^{2}\right)$} & \multirow{2}{*}{\multicolumn{2}{|c|}{$2 p_{1}$}} \\
\hline & \multicolumn{2}{|c|}{ is } & \multicolumn{2}{|c|}{$2 s$} & \multicolumn{2}{|c|}{$2 p_{0}$} & & \\
\hline & FBA & DWBA & FBA & DWBA & FBA & DWBA & FBA & DWBA \\
\hline 20 & 10.505 & 10.281 & 0.7149 & 0.8598 & 0.5206 & 0.35 & $0.893[-1]$ & $0.524[-1]$ \\
\hline 30 & 5.182 & 5.226 & 0.5816 & 0.644 & 0.2367 & 0.1678 & $0.600[-1]$ & $0.397[-1]$ \\
\hline 40 & 2.664 & 2.75 & 0.3514 & 0.3806 & 0.1182 & $0.926[-1]$ & $0.306[-1]$ & $0.215[-1]$ \\
\hline 50 & 1.460 & 1.538 & 0.2063 & 0.2223 & $0.615[-1]$ & $0.518[-1]$ & $0.153[-1]$ & $0.111[-1]$ \\
\hline 60 & 0.8463 & 0.9082 & 0.1236 & 0.1333 & $0.333[-1]$ & $0.295[-1]$ & $0.789[-2]$ & $0.589[-2]$ \\
\hline 75 & 0.4078 & 0.4487 & $0.608[-1]$ & $0.660[-1]$ & $0.143[-1]$ & $0.134[-1]$ & $0.317[-2]$ & $0.243[-2]$ \\
\hline 100 & 0.1443 & 0.1646 & $0.215[-1]$ & $0.239[-1]$ & $0.415[-2]$ & $0.422[-2]$ & $0.849[-3]$ & $0.670[-3]$ \\
\hline 125 & $0.600[-1]$ & $0.706[-1]$ & $0.887[-2]$ & $0.101[-1]$ & $0.144[-2]$ & $0.156[-2]$ & $0.278[-3]$ & $0.224[-3]$ \\
\hline 150 & $0.281[-1]$ & $0.339[-1]$ & $0.409[-2]$ & $0.475[-2]$ & $0.576[-3]$ & $0.658[-3]$ & $0.106[-3]$ & $0.868[-4]$ \\
\hline 175 & $0.144[-1]$ & $0.178[-1]$ & $0.207[-2]$ & $0.246[-2]$ & $0.256[-3]$ & $0.307[-3]$ & $0.452[-4]$ & $0.377[-4]$ \\
\hline 200 & $0.789[-2]$ & $0.996[-2]$ & $0.112[-2]$ & $0.136[-2]$ & $0.124[-3]$ & $0.155[-3]$ & $0.212[-4]$ & $0.180[-4]$ \\
\hline 225 & $0.459[-2]$ & $0.590[-2]$ & $0.641[-3]$ & $0.798[-3]$ & $0.642[-4]$ & $0.836[-4]$ & $0.107[-4]$ & $0.921[-5]$ \\
\hline 250 & $0.279[-2]$ & $0.365[-2]$ & $0.386[-3]$ & $0.491[-3]$ & $0.353[-4]$ & $0.476[-4]$ & $0.576[-5]$ & $0.501[-5]$ \\
\hline 275 & $0.177[-2]$ & $0.235[-2]$ & $0.241[-3]$ & $0.313[-3]$ & $0.203[-4]$ & $0.283[-4]$ & $0.326[-5]$ & $0.287[-5]$ \\
\hline 300 & $0.116[-2]$ & $0.156[-2]$ & $0.156[-3]$ & $0.207[-3]$ & $0.122[-4]$ & $0.175[-4]$ & $0.193[-5]$ & $0.171[-5]$ \\
\hline 325 & $0.780[-3]$ & $0.107[-2]$ & $0.104[-3]$ & $0.141[-3]$ & $0.761[-5]$ & $0.112[-4]$ & $0.118[-5]$ & $0.106[-5]$ \\
\hline 350 & $0.539[-3]$ & $0.745[-3]$ & $0.715[-4]$ & $0.981[-4]$ & $0.489[-5]$ & $0.738[-5]$ & $0.748[-6]$ & $0.680[-6]$ \\
\hline 375 & $0.381[-3]$ & $0.532[-3]$ & $0.501[-4]$ & $0.698[-4]$ & $0.323[-5]$ & $0.498[-5]$ & $0.488[-6]$ & $0.447[-6]$ \\
\hline 400 & $0.275[-3]$ & $0.387[-3]$ & $0.358[-4]$ & $0.507[-4]$ & $0.218[-5]$ & $0.344[-5]$ & $0.326[-6]$ & $0.302[-6]$ \\
\hline 425 & $0.201[-3]$ & $0.287[-3]$ & $0.260[-4]$ & $0.374[-4]$ & $0.151[-5]$ & $0.242[-5]$ & $0.223[-6]$ & $0.208[-6]$ \\
\hline 450 & $0.150[-3]$ & $0.215[-3]$ & $0.193[-4]$ & $0.281[-4]$ & $0.106[-5]$ & $0.173[-5]$ & $0.155[-6]$ & $0.146[-6]$ \\
\hline 475 & $0.113[-3]$ & $0.164[-3]$ & $0.145[-4]$ & $0.213[-4]$ & $0.760[-6]$ & $0.126[-5]$ & $0.110[-6]$ & $0.104[-6]$ \\
\hline 500 & $0.868[-4]$ & $0.127[-3]$ & $0.110[-4]$ & $0.164[-4]$ & $0.553[-6]$ & $0.932[-6]$ & $0.795[-7]$ & $0.758[-7$ \\
\hline
\end{tabular}

mer $^{17}$ using the first-order Faddeev-Watson multiplescattering approximation, Banerji et al. ${ }^{18}$ using the Faddeev formalism, Mandal et al. ${ }^{19}$ using an approximate form of the Faddeev equations, Basu et al. ${ }^{20}$ using the close-coupling approximation, Drachman et al. ${ }^{21}$ using the partial-wave method, Mandal and $G u^{22} a^{22}$ using the first Born, the first-order exchange, and the modified Born approximations, Mandal et al. ${ }^{23}$ using the distorted-wave Born approximation, Sil et al. ${ }^{24}$ using the first Born approximation for excited-state Ps formation, Khan et al. ${ }^{25,26}$ using the distorted-wave approximation in combination with the polarized-orbital method, Saha and $\mathrm{Roy}^{27}$ using the first Born and the first-order exchange approximations, McGuire et al. ${ }^{14}$ using the Born approximation complete through second order in the collisional potentials, Straton ${ }^{28}$ using the Fock-Tani transformations, and Basu and Ghosh ${ }^{15}$ using the second Born approximation. A comparison of the present integrated cross sections for Ps formation at various impact energies has been made in Table II with other theoretical investigations. The present values for the cross sections differ from those of other investigations due to different approximations of various methods, yet the present values are comparable and consistent with the values from most of the other calculations. From Table II, it can be seen that the present values are more consistent with those obtained using the first-order exchange approximation ${ }^{27}$ at lower energies than with those obtained using the

TABLE II. Comparison of the present integrated cross sections (in units of $a_{0}^{2}$ ) for the positronium formation in the ground and excited states with other calculated values. The notation $a[b]$ means $a \times 10^{b}$.

\begin{tabular}{|c|c|c|c|c|c|c|c|c|c|}
\hline $\begin{array}{c}E \\
(\mathrm{eV}) \\
\end{array}$ & Present & $\begin{array}{c}\text { Ref. } \\
15\end{array}$ & $\begin{array}{c}\text { is (units of } a \\
\text { Ref. } \\
27\end{array}$ & $\begin{array}{c}\text { Ref. } \\
23\end{array}$ & $\begin{array}{c}\text { Ref. } \\
28\end{array}$ & Present & $\begin{array}{c}\sigma_{2(s+p)}(u \\
\text { Ref. } \\
26\end{array}$ & $\begin{array}{c}\text { dits of } a_{0}^{2} \text { ) } \\
\text { Ref. } \\
15\end{array}$ & $\begin{array}{c}\text { Ref. } \\
27\end{array}$ \\
\hline 20 & 10.28 & & 10.63 & 10.34 & 11.36 & 1.315 & 1.087 & & 1.116 \\
\hline 30 & 5.23 & & 5.59 & & 5.55 & 0.891 & 0.763 & & 0.855 \\
\hline 40 & 2.75 & & & & 2.83 & 0.516 & & & \\
\hline 50 & 1.54 & 1.932 & 1.71 & 1.60 & 1.53 & 0.296 & 0.241 & 0.633 & 0.313 \\
\hline 100 & 0.165 & 0.165 & 0.192 & 0.180 & 0.147 & $0.295[-1]$ & $0.330[-1]$ & $0.458[-1]$ & $0.342[-1]$ \\
\hline 200 & $0.996[-2]$ & $0.983[-2]$ & $0.120[-1]$ & $0.120[-1]$ & & $0.155[-2]$ & & $0.179[-2]$ & $0.192[-2]$ \\
\hline 300 & $0.156[-2]$ & $0.153[-2]$ & & & & $0.228[-3]$ & & $0.240[-3]$ & \\
\hline 500 & $0.127[-3]$ & $0.121[-3]$ & $0.154[-3]$ & & $0.858[-4]$ & $0.175[-4]$ & & $0.179[-4]$ & $0.219[-4]$ \\
\hline
\end{tabular}


TABLE III. The total cross sections (in units of $a_{0}^{2}$ ), $\sigma^{T}$, in the FBA and DWBA for the formation of positronium from hydrogen atoms at various positron impact energies. The notation $a[b]$ means $a \times 10^{b}$.

\begin{tabular}{|c|c|c|c|c|c|}
\hline \multirow{2}{*}{$\begin{array}{c}E \\
(\mathrm{eV}) \\
\end{array}$} & \multicolumn{2}{|c|}{$\sigma^{T}$ (units of $a_{0}^{2}$ ) } & \multirow{2}{*}{$\begin{array}{c}E \\
(\mathrm{eV})\end{array}$} & \multicolumn{2}{|c|}{$\sigma^{r}$ (units of $a_{0}^{2}$ ) } \\
\hline & FBA & DWBA & & FBA & DWBA \\
\hline 20 & 12.791 & 12.406 & 250 & $0.349[-2]$ & $0.454[-2]$ \\
\hline 30 & 6.699 & 6.666 & 275 & $0.220[-2]$ & $0.291[-2]$ \\
\hline 40 & 3.522 & 3.584 & 300 & $0.144[-2]$ & $0.193[-2]$ \\
\hline 50 & 1.942 & 2.017 & 325 & $0.965[-3]$ & $0.131[-2]$ \\
\hline 60 & 1.125 & 1.190 & 350 & $0.655[-3]$ & $0.918[-3]$ \\
\hline 75 & 0.5393 & 0.585 & 375 & $0.469[-3]$ & $0.655[-3]$ \\
\hline 100 & 0.1886 & 0.2121 & 400 & $0.337[-3]$ & $0.476[-3]$ \\
\hline 125 & $0.776[-1]$ & $0.901[-1]$ & 425 & $0.247[-3]$ & $0.352[-3]$ \\
\hline 150 & $0.360[-1]$ & $0.430[-1]$ & 450 & $0.183[-3]$ & $0.264[-3]$ \\
\hline 175 & $0.183[-1]$ & $0.224[-1]$ & 475 & $0.138[-3]$ & $0.201[-3]$ \\
\hline 200 & $0.997[-2]$ & $0.125[-1]$ & 500 & $0.106[-3]$ & $0.155[-3]$ \\
\hline 225 & $0.576[-2]$ & $0.735[-2]$ & & & \\
\hline
\end{tabular}

second-order Born approximation ${ }^{15}$ at higher energies for both the ground- and the excited-state formations. For the ground-state Ps formation, the distorted-wave Bornapproximation values of Mandal et al. ${ }^{23}$ are a little higher than the present values, while for the excited-state Ps formation the distorted-wave Born-approximation values of Khan et al. $^{26}$ are a little smaller than the present values, except at $100 \mathrm{eV}$. Perhaps, a better judge-

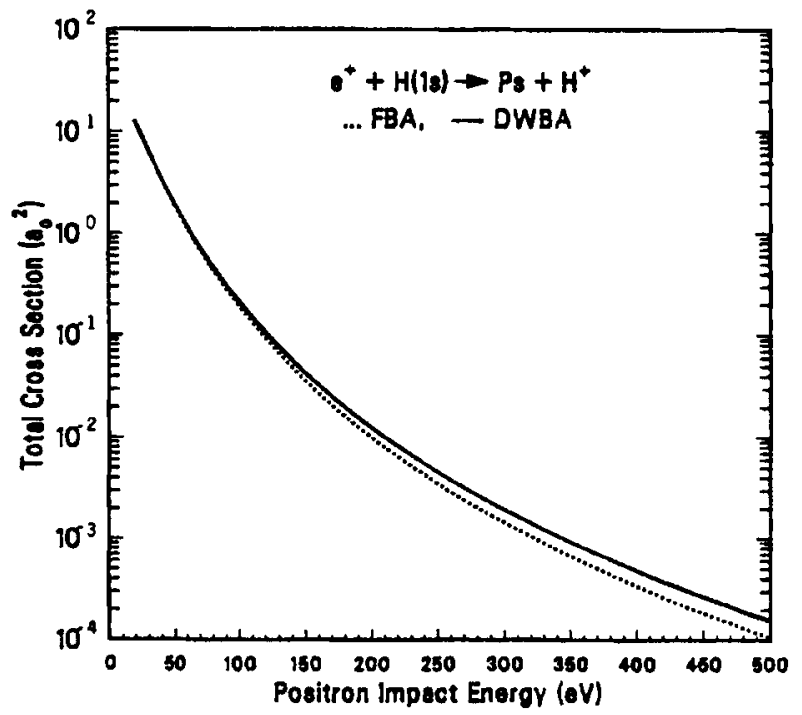

FIG. 6. Total integrated cross sections for positronium formation at various energies. The solid curve corresponds to the DWBA and the dotted curve to the FBA. ment among these values using various methods could be made after some experimentally measured values of Ps formation are obtained.

Table III presents the total cross sections, using the $1 / n^{3}$ behavior, for positronium formation from an hydrogen atom over the incident-positron energy range 20-500 $\mathrm{eV}$. The total cross section using the DWBA is higher than that using the FBA in the energy range $40-500 \mathrm{eV}$, but lower than that using the FBA at 20 and $30 \mathrm{eV}$. This is also evident in Fig. 6. The convergence at somewhat lower energies and then divergence at higher energies between the integrated cross sections using the FBA and DWBA has been observed in other methods as well. This shows that the present distorting potential has not lost its effect on the capture process significantly in the energy range considered. This is not unusual for a hard-type distortion potential ${ }^{29}$ which continues to exert influence in the high-energy limit, providing a continuous finite difference between the FBA and the DWBA cross sections.

In conclusion, the cross sections for the formation of positronium in the ground $(1 s)$ and in the excited states $\left(2 s, 2 p_{0}\right.$, and $\left.2 p_{ \pm 1}\right)$ as well as the total cross sections for the formation of $P s$ in all states by the impact of intermediate-energy $(20-500 \mathrm{eV})$ positrons on hydrogen atoms have been calculated using the first Born and the distorted-wave Born approximations. The present results using the FBA agree with those carried out by other investigators and the results using the DWBA are comparable to those obtained using various other methods.

\section{ACKNOWLEDGMENTS}

I would like to express my sincere gratitude to Professor J. M. Wadehra for much helpful information and discussions. 
${ }^{1}$ M. Deutsch, Prog. Nucl. Phys. 3, 131 (1953).

${ }^{2}$ H. S. W. Massey and C. B. O. Mohr, Proc. Phys. Soc. London, Sect. A 67, 695 (1954).

${ }^{3}$ S. L. Varghese, E. S. Ensberg, V. W. Hughes, and I. Lindgren, Phys. Lett. A 49, 415 (1974).

${ }^{4}$ K. F. Canter, A. P. Mills, Jr., and S. Berko, Phys. Rev. Lett. 34, 177 (1975).

${ }^{5}$ A. P. Mills, Jr., S. Berko, and K. F. Canter, Phys. Rev, Lett. 34, 1541 (1975); S. Hatamian, R. S. Conti, and A. Rich, ibid. 58, 1833 (1987).

${ }^{6}$ M. A. Stroscio, Phys. Rep. 22, 215 (1975).

7J. R. Oppenheimer, Phys. Rev. 31, 349 (1928).

${ }^{8}$ H. C. Brinkman and H. A. Kramers, Proc. Acad. Sci. Amsterdam 33, 973 (1930).

${ }^{9}$ R. H. Bassel and E. Gerjuoy, Phys. Rev. 117, 749 (1960).

${ }^{10}$ R. Shakeshaft and J. M. Wadehra, Phys. Rev. A 22, 968 (1980); S. N. Nahar and J. M. Wadehra, ibid. 35, 4533 (1987).

"Sultana N. Nahar and J. M. Wadehra, Phys. Rev. A 37, 4118 (1988).

${ }^{12}$ K. Omidvar, Phys. Rev. A 12, 911 (1975).

${ }^{13}$ J. R. Macdonald, P. Richard, C. L. Cocke, M. Brown, and 1. A. Sellin, Phys. Rev. Lett. 31, 684 (1973).

14J. H. McGuire, N. C. Sil, and N. C. Deb, Phys. Rev. A 34, 685 (1986); N. C. Deb, J. H. McGuire, and N. C. Sil, ibid. 36, 3707
(1987).

${ }^{15}$ M. Basu and A. S. Ghosh, J. Phys. B 21, 3439 (1988).

16I. M. Cheshire, Proc. Phys. Soc. London 83, 227 (1964).

17J. C. Y. Chen and P. J. Kramer, Phys. Rev. A 5, 1207 (1972).

${ }^{18} \mathrm{G}$. Banerji, A. S. Ghosh, and N. C. Sil, Phys. Rev. A 7, 571 (1973).

${ }^{19}$ P. Mandal, B. C. Saha, and N. C. Sil, J. Chem. Phys. 63, 4767 (1975).

${ }^{20}$ D. Basu, G. Banerji, and A. S. Ghosh, Phys. Rev. A 13, 1381 (1976).

${ }^{21}$ R. J. Drachman, K. Omidvar, and J. H. McGuire, Phys. Rev. A 14, 100 (1976).

22P. Mandal and S. Guha, J. Phys. B 12, 1603 (1979).

23P. Mandal, S. Guha, and N. C. Sil, J. Phys. B 12, 2913 (1979).

${ }^{24}$ N. C. Sil, B. S. Saha, H. P. Saha, and P. Mandal, Phys. Rev. A 19, 655 (1979).

${ }^{25}$ P. Khan and A. S. Ghosh, Phys. Rev, A 27, 1904 (1983).

26P. Khan, P. S. Mazumdar, and A. S. Ghosh, Phys. Rev. A 31, 1405 (1985).

${ }^{27}$ B. C. Saha and P. K. Roy, Phys. Rev. A 30, 2980 (1984).

28J. C. Straton, Phys. Rev. A 35, 3725 (1987) and private communication.

${ }^{29}$ N. Toshima, T. Ishihara, and J. Eichler, Phys. Rev. A 36, 2659 (1987). 